\title{
Modern landscape design under the influence of eco ISM
}

\author{
MA Dongqing ${ }^{1, \text { a }}$ \\ ${ }^{1}$ Dongseo University, 47011, Korea \\ amadongqing@126.com
}

Keywords: Ecology; modern landscape design; environmental ecology.

\begin{abstract}
With the development of society, people in the landscape design requirements are also gradually improve, between the new design concept and construction of the landscape both needs to be effective with attention to the landscape design under the current social development needs. The concept of sustainable development, which has been pursued in the process of social development, has brought about a series of changes in people's lives, and there has been a more profound understanding of ecology. Therefore, in the process of modern landscape construction, to continue the introduction of the concept of sustainable development, as the core concept of landscape construction, adhere to the ecological doctrine, in order to further promote the construction of ecological landscape, highlighting contemporary ecological humanism. Based on this, this paper aims at the current ecological landscape design of contemporary society related content, in order to be able to provide a theoretical basis for the modern landscape design.
\end{abstract}

\section{Introduction}

Landscape design has become a modern problem that has been widely discussed in the process of social development. With the development of society, the process has evolved so far. But in today's society because of the increasingly serious social problems [1], for the construction of modern landscape makes people combine between ecological values and the landscape design of the two, is an inevitable trend of the current society [2]. In the natural environment, there is a strong link between topographic features of modern landscape and the part of the humanism from the ecological perspective, the modern landscape construction in regional culture inheritance of each region, and sustainable development is extremely scarce. So how in the modern landscape ecological design process for efficient integration with the modern landscape, how under the ecological thought in effect, make the impetus to modern landscape construction, improve the city landscape environment, based on the concept of sustainable development, to improve the quality of life in the city. This paper studies the problems related to modern landscape design under the influence of ecology, so as to promote the continuous development of society.

\section{The concept of modern landscape ecological design}

In the process of social development, for landscape construction based on the concept of ecological Marxism, is the current city development process to achieve the ecological planning of a large part of [3], at the same time, emphasizing the ecological construction of modern landscape concept, it is people-oriented, ecological sustainable development is the main idea of construction. The construction of modern landscape under the influence of eco ism should not only follow the slogan of ecology, but put the idea into the whole process of landscape construction. The ecological landscape is a kind of development concept to promote the sustainable development of construction, and it is also the slogan and goal of promoting the development of the whole city. The ecological landscape design to landscape, realize the efficient integration of humanistic spirit and ecological spirit, solve the relationship between humanity and nature, which combine to enhance the entire city cultural heritage and environmental quality of life [4]. At the same time the ecological landscape 
construction should also be on regional characteristics and energy utilization aspects of considering many factors, make full use of the location, so as to build a harmonious ecological landscape design, ecological benefit real play.

\section{The regional characteristic ecotype landscape design idea difference formation}

\section{The reasons for the formation of regional ecological ideas}

The so-called ecological concept and landscape design with regional characteristics, its regional characteristics are often related to the geographical features of the landscape and the humanistic spirit of the region. This includes landscape area location, geographical conditions, weather factors, water quality, climate and plant resources and regional geology; historical origin, the region is rich in cultural values, and people's life behavior. The characteristics of regional landscape ecological construction is often includes two aspects: one is for the position of environmental features in the landscape (such as landscape location, climate resources, etc.); on the other hand is the humanistic spirit of the landscape, which also contains a historical and cultural heritage in the region, the region is also the human consciousness of culture.

\section{Regional characteristics and cultural characteristics of the regional characteristics of the differences}

The ecological landscape construction has nothing to do with its own geographical features, thus creating ecological landscape construction of different regions and different features (5). In the process of design, the landscape and the geographical, historical, cultural and natural and human elements in the ecological concept are reflected in the landscape construction under the ecological concept. The landscape construction has different geographical features, which bring up the geographical features and cultural characteristics of different landscape designs under the influence of ecological ideas. By taking the landscape design of the different aesthetic characteristics of space as well as the integration of local history and cultural background, so as to carry out the integration of multiple levels, thus the construction of ecological landscape with regional characteristics. China's vast territory, because of its geographical location and historical development of human differences, there are significant regional differences. While residential construction should make full use of the regional characteristics, to design a different geographical characteristics of the landscape, the topographic features at the same time can also be the geographical location and the fusion, the shape of landscape ecological concept very geographical features [6] creative. The regional name exists in certain specific areas, because of the different geographical and cultural, thus showing different features in the concept of ecological landscape design, and geographical and cultural background as well as many different factors for regional value makes all the residential landscape style is different, which had very geographical features the concept of ecological landscape design.

\section{The ecological concept of landscape construction and the root causes of the solution}

\section{The current landscape construction concept is a single root}

At present in our country due to the process of social development is too fast, resulting in the landscape construction process exists in the homogeneous design concept is single, the design of texture is low, the vast majority of landscape design in the blind copy, which the design of popular design, which refers to the vane design, the landscape construction of the current lack of the lack of affinity characteristics. The reason for this phenomenon mainly has two aspects: one is because now the vast majority of the landscape construction company is designed based on the purpose of obtaining benefits, to meet customer demands, will not show the characteristic on the first design, landscape design to make uniform follow no emotional content design characteristics, causes now countless similar landscape design; on the other hand is due to the vast majority of the landscape designers before residential landscape design, no systematic comprehensive understanding of 
landscape location and geographical features of landscape not high degree of attention, cognition on regional geographical and cultural features are too simple. Therefore, in the design process due to the lack of awareness of the landscape design, there will be one-sided greening rate equivalent design of high rate of quality, as well as the north and South Regional plants affected the probability of confusion, the survival of plants, more is not conducive to the ecological construction of landscape.

\section{The current ecological concept of landscape design solutions}

Landscape design is to solve the problem of people's life and work there, under the concept of ecological landscape design reflects the ecological characteristics of landscape location, to the residents to facilitate life but also has regional characteristics, in addition, combined with the natural landscape, integration of regional location, so as to promote a the development process of landscape design.

\section{Respects the natural environment of the ecological landscape}

The natural environment of the landscape has a great influence on the landscape construction, and the natural environment is enough to change the overall ecological features of the ecological landscape, so it is the root of the landscape design idea. In the course of design, the geographical features of the landscape are shown mainly from the geographical location, natural environment, climate and plant aspects of the landscape. [7]. The regional landscape should keep the geography features originally existed, and improve on the basis of a reservation, pay attention to the geographical landscape shape changes with the terrain, ecological landscape design creative personalized. Each region has a natural climate gap between different climate, different geological hydrology, for the region's people's living conditions and lifestyle will have an impact on the landscape, regional style will have a direct impact. Only the local conditions [8], the landscape construction of ecological concept of geographical location for a detailed understanding, which were suitable for the growth of the local plants, improve the survival rate of plants, not only can reduce the maintenance costs, the most important is to show the landscape ecological philosophy also can show the regional characteristics of landscape.

\section{Respects the humanistic spirit of the ecological landscape}

In the process of landscape design under the ecological concept, in order to avoid the monotonous appearance of the landscape design and the empty content, we should pay attention to the humanistic spirit of the landscape [9]. In today's society, the concept of ecological landscape design ideas included in the development of the traditional history and culture, the ecological landscape design and the continuation of the traditional humanistic spirit, in the design process, with the help of ecological landscape performance of the regional culture, respect between different regions of Humanities and cultural diversity. Landscape design and regional ecology under the concept of culture are inextricably linked, reflect the geographical and cultural characteristics, landscape design, cultural characteristics, and ecological concept, thus forming a unique ecological landscape style geographical features. For example, in the design process, the rational use of regional cultural products, so that the ecological landscape to become people's spiritual support.

\section{Ecological landscape design method and its application}

The ecological landscape should be flexibly used in the design process, so as to construct a diversified and characteristic ecological landscape. Therefore, it is necessary to pay attention to every link of the landscape ecological concept design process, so as to promote the construction of ecological landscape.

\section{Pay attention to combining topographic elements}

In the process of design and construction of the ecological landscape, will inevitably though and location of the terrain topography combines a variety of available elements will be included, will give full consideration to the animal and human living space etc.. At the same time but also consider the landscape noise control, such as the construction of the landscape in the coastal city, then we must 
take into account the design problem of wetland landscape in the construction of ecological landscape, full consideration for the preservation and control of water, although in the attention of terrain features, construction characteristics of the ecological landscape [for 10].

\section{Plant elements}

In the process of the construction of ecological landscape, the most important and essential elements is plant elements, through the rational use of plant elements in the ecological landscape design of the current, can promote the construction of the concept of ecological landscape. Therefore, plants play a crucial role in the construction of landscape under the influence of the ecological concept. Landscape designers need to attach great importance to the early design, and scientifically select plants. We should consider the characteristics of the region, select the native plants, and pay attention to the visual appreciation after planting. The plants of each ecological community should be reflected by the scientific and normative design methods, and the rational and normative ecological plant communities should be constructed to promote the construction of ecological landscape.

\section{Summary}

In the new era of diversification of people continue to pursue things, for the landscape design process to ecological rational use, based on the consideration of design style, combining regional, plant elements and spiritual factors, pay attention to ecological concept into the landscape design, so as to promote the ecological the landscape design of the road of sustainable development. Combining ecology with landscape construction will promote the sustainable development of the whole city.

\section{Acknowledgements}

This research was supported by BK21plus Level-up Project for Marine design team in Dongseo University.

\section{References}

[1] Liu Changdong. Modern urban landscape design under eco ism [J]. building materials and decoration, 2017, (08): 68-69.

[2] Li Jiangshan, Zheng Shaojiang. Post industrial landscape design language influenced by postmodernism. [J]. popular literature and art, 2016, (11): 68.

[3] Li Jing. Research on the forms of modern landscape design under the influence of ecological science [J]. architectural knowledge, 2016, (04): 32.

[4] Wang Bin. Research on the influence of postmodernism design trend on contemporary landscape architecture [D]. Dalian University of technology, 2014.

[5] Zhao Chenyang. Modern landscape design under the influence of ecological science [D]. Nanjing Forestry University, 2005.

[6] Dou Yinghui. Interpretation of the poetic landscape of ecological landscape. Also on the influence of ecological ethics on modern landscape design [J]. beauty and time, 2010 (1): 81-83.

[7] door opening. Development trend of modern landscape design [J]. large stage, 2012 (3): 135-135.

[8] Ge Ping, Wang Shudong. Study on Landscape Design -- Taking Zhongshan Qijiang Park as an example of $[\mathrm{J}]$. Chinese environmental management from the viewpoint of environment protection and ecology, 2010 (3): 58-60.

[9] Ren Junjun. By [J]. of Hubei University of Economics's ecological thoughts in Modern Landscape Design Journal (SOCIAL SCIENCE EDITION), 2012, 9 (8): 28-29. 
[10] Wang Limin. Analysis of the modern landscape design and landscape art [J]. management and technology of small and medium-sized enterprises management, 2014 (7): 192-193. 